\title{
Multiple Soliton Solutions of the Sawada-Kotera Equation with a Nonvanishing Boundary Condition and the Perturbed Korteweg de Vries Equation by Using the Multiple Exp-Function Scheme
}

\author{
Abdullahi Rashid Adem, ${ }^{1}$ Mohammad Mirzazadeh,, Qin Zhou ${ }^{2},{ }^{3}$ and Kamyar Hosseini ${ }^{4}{ }^{4}$ \\ ${ }^{1}$ Department of Mathematical Sciences, North-West University, Private Bag X 2046, Mmabatho 2735, South Africa \\ ${ }^{2}$ Department of Engineering Sciences, Faculty of Technology and Engineering, East of Guilan, University of Guilan, \\ Rudsar 44891-63157, Iran \\ ${ }^{3}$ School of Electronics and Information Engineering, Wuhan Donghu University, Wuhan 430212, China \\ ${ }^{4}$ Department of Mathematics, Rasht Branch, Islamic Azad University, Rasht, Iran
}

Correspondence should be addressed to Qin Zhou; qinzhou@whu.edu.cn

Received 16 January 2019; Accepted 14 March 2019; Published 2 May 2019

Academic Editor: Boris G. Konopelchenko

Copyright (c) 2019 Abdullahi Rashid Adem et al. This is an open access article distributed under the Creative Commons Attribution License, which permits unrestricted use, distribution, and reproduction in any medium, provided the original work is properly cited.

\begin{abstract}
The Sawada-Kotera equation with a nonvanishing boundary condition, which models the evolution of steeper waves of shorter wavelength than those depicted by the Korteweg de Vries equation, is analyzed and also the perturbed Korteweg de Vries (pKdV) equation. For this goal, a capable method known as the multiple exp-function scheme (MEFS) is formally utilized to derive the multiple soliton solutions of the models. The MEFS as a generalization of Hirota's perturbation method actually suggests a systematic technique to handle nonlinear evolution equations (NLEEs).
\end{abstract}

\section{Introduction}

In the applied science, NLEEs are extensively used in theoretical studies to model a wide range of nonlinear phenomena. To comprehend the mechanisms of nonlinear phenomena, it is vital to investigate the solutions of NLEEs [1-15]. One specific tool that has recently achieved a special interest from academic researchers is the multiple exp-function scheme $[16,17]$. The MEFS supposes that the multisoliton solutions of NLEEs can be presented as $u(x, t)=p / q$ in which $p$ and $q$ are polynomials of exponential functions. The SK equation with a nonvanishing boundary condition $[18,19]$

$$
\begin{aligned}
u_{t}+ & a\left(\frac{a}{5 b} u+3 u^{2}+u_{x x}\right)_{x} \\
& +b\left(15 u^{3}+15 u u_{x x}+u_{x x x x}\right)_{x}=0
\end{aligned}
$$

is one of NLEEs that models the evolution of steeper waves of shorter wavelength than those explained by the KdV equation and its perturbed form. By using the binary-Bell-polynomial Hirota method and symbolic computation, the bilinear form and $\mathrm{N}$-soliton solutions for this model were derived in [19]. The perturbed form of KdV equation [18, 20-22]

$$
\begin{aligned}
u_{t}+ & 6 u u_{x}+u_{x x x} \\
& +\epsilon\left(30 u^{2} u_{x}+10 u u_{x x x}+20 u_{x} u_{x x}+u_{x x x x x}\right) \\
& =0,
\end{aligned}
$$

is another kind of NLEEs describing some arrays of wave crests. The bilinear form, Bäcklund transformation, superposition formulae, and $\mathrm{N}$-soliton solutions in terms of the Wronskian were done in [22].

For computational purposes, we use the transformation

$$
u=v_{x}
$$


to convert (1) and (2) into the following, respectively:

$$
\begin{aligned}
v_{x t} & +a\left(\frac{a}{5 b} v_{x x}+6 v_{x} v_{x x}+v_{x x x x}\right) \\
& +b\left(45 v_{x}^{2} v_{x x}+15 v_{x} v_{x x x x}+15 v_{x x} v_{x x x}+v_{x x x x x x}\right) \\
= & 0
\end{aligned}
$$

and

$$
\begin{aligned}
v_{x t} & +6 v_{x} v_{x x}+v_{x x x x} \\
& +\epsilon\left(30 v_{x}^{2} v_{x x}+10 v_{x} v_{x x x x}+20 v_{x x} v_{x x x}+v_{x x x x x x}\right) \\
= & 0 .
\end{aligned}
$$

The key goal of present work is applying the MEFS to generate the multiple soliton solutions of the models (1) and (2).

\section{Multiple Exp-Function Method}

The key steps of MEFS can be summarized as follows [16, 17].

Step 1. Let us consider the following $(1+1)$-dimensional NLEE:

$$
P\left(x, t, u_{x}, u_{t}, \cdots\right)=0 .
$$

Step 2. Suppose the solution of above NLEE can be expressed as

$$
\begin{array}{r}
u(x, t)=\frac{p\left(\eta_{1}, \eta_{2}, \cdots, \eta_{n}\right)}{q\left(\eta_{1}, \eta_{2}, \cdots, \eta_{n}\right)}, \\
p=\sum_{r, s=1}^{n} \sum_{i, j=0}^{M} p_{r s, i j} \eta_{r}^{i} \eta_{s}^{j}, \\
q=\sum_{r, s=1}^{n} \sum_{i, j=0}^{N} q_{r s, i j} \eta_{r}^{i} \eta_{s}^{j},
\end{array}
$$
and

in which $p_{r s, i j}$ and $q_{r s, i j}$ are unknowns to be determined

$$
\begin{aligned}
\eta_{i} & =c_{i} e^{\xi_{i}}, \\
\xi_{i} & =k_{i} x-\omega_{i} t, \\
& 1 \leqslant i \leqslant n,
\end{aligned}
$$

Step 3. Substituting (7) and its derivatives into (6) yields the following transformed equation:

$$
Q\left(x, t, \eta_{1}, \eta_{2}, \cdots, \eta_{n}\right)=0 .
$$

Step 4. By setting the numerator of the function $Q\left(x, t, \eta_{1}, \eta_{2}\right.$, $\left.\cdots, \eta_{n}\right)$ to zero, we will reach an algebraic system which its solution yields the multiple wave solution of (6) as

$$
u(x, t)=\frac{p\left(c_{1} e^{k_{1} x-\omega_{1} t}, \cdots, c_{n} e^{k_{n} x-\omega_{n} t}\right)}{q\left(c_{1} e^{k_{1} x-\omega_{1} t}, \cdots, c_{n} e^{k_{n} x-\omega_{n} t}\right)} .
$$

2.1. Multiple Soliton Solutions of SK Equation with a Nonvanishing Boundary Condition. In the current subsection, the multiple soliton solutions of SK equation with a nonvanishing boundary condition are derived through the MEFS.

2.1.1. One-Soliton Solution of (1). To obtain one-soliton solution, it is assumed

$$
\begin{aligned}
v(x, t) & =\frac{p}{q}, \\
p & =A_{1} \mathrm{e}^{\theta_{1}}, \\
q & =1+\mathrm{e}^{\theta_{1}},
\end{aligned}
$$

where $A_{1}$ is a constant and

$$
\theta_{1}=k_{1} x-\omega_{1} t
$$

the dispersion is

$$
\omega_{1}=\frac{k_{1}\left(5 b^{2} k_{1}^{4}+5 a b k_{1}^{2}+a^{2}\right)}{5 b} .
$$

Now, applying the MEFS results in

$$
A_{1}=2 k_{1}
$$

one-soliton solution can be presented as

$$
v(x, t)=\frac{2 k_{1} \mathrm{e}^{k_{1} x-\omega_{1} t}}{1+\mathrm{e}^{k_{1} x-\omega_{1} t}}
$$

where $k_{1}$ is arbitrary but $\omega_{1}$ is defined by (13).

2.1.2. Two-Soliton Solution of (1). To seek two-soliton solution, the following ansatz is considered

$$
v(x, t)=\frac{p}{q}
$$

in which $p$ and $q$ are defined as

$$
\begin{aligned}
p= & 2 k_{1} \mathrm{e}^{k_{1} x-\omega_{1} t}+2 k_{2} \mathrm{e}^{k_{2} x-\omega_{2} t} \\
& +2 A_{12}\left(k_{1}+k_{2}\right) \mathrm{e}^{k_{1} x-\omega_{1} t} \mathrm{e}^{k_{2} x-\omega_{2} t}, \\
q= & 1+\mathrm{e}^{k_{1} x-\omega_{1} t}+\mathrm{e}^{k_{2} x-\omega_{2} t}+A_{12} \mathrm{e}^{k_{1} x-\omega_{1} t} \mathrm{e}^{k_{2} x-\omega_{2} t} .
\end{aligned}
$$

Now, by applying the MEFS, we acquire 


$$
\begin{aligned}
& A_{12}=\frac{5 b k_{1}{ }^{4}-15 b k_{1}{ }^{3} k_{2}+20 b k_{1}{ }^{2} k_{2}{ }^{2}-15 b k_{1} k_{2}{ }^{3}+5 b k_{2}{ }^{4}+3 a k_{1}{ }^{2}-6 a k_{1} k_{2}+3 a k_{2}{ }^{2}}{5 b k_{1}{ }^{4}+15 b k_{1}{ }^{3} k_{2}+20 b k_{1}{ }^{2} k_{2}{ }^{2}+15 b k_{1} k_{2}{ }^{3}+5 b k_{2}{ }^{4}+3 a k_{1}{ }^{2}+6 a k_{1} k_{2}+3 a k_{2}{ }^{2}} \text {, } \\
& \omega_{1}=\frac{k_{1}\left(5 b^{2} k_{1}^{4}+5 a b k_{1}^{2}+a^{2}\right)}{5 b} \text {, } \\
& \omega_{2}=\frac{k_{2}\left(5 b^{2} k_{2}{ }^{4}+5 a b k_{2}^{2}+a^{2}\right)}{5 b} .
\end{aligned}
$$

2.1.3. Three-Soliton Solution of (1). To derive three-soliton solution, it is assumed

$$
v(x, t)=\frac{p}{q}
$$

in which $p$ and $q$ are defined as

$$
\begin{aligned}
p & =2 k_{1} \mathrm{e}^{k_{1} x-\omega_{1} t}+2 k_{2} \mathrm{e}^{k_{2} x-\omega_{2} t}+2 k_{3} \mathrm{e}^{k_{3} x-\omega_{3} t} \\
& +2 A_{12}\left(k_{1}+k_{2}\right) \mathrm{e}^{-\omega_{1} t+k_{1} x} \mathrm{e}^{-\omega_{2} t+k_{2} x} \\
& +2 A_{13}\left(k_{1}+k_{3}\right) \mathrm{e}^{k_{1} x-\omega_{1} t} \mathrm{e}^{k_{3} x-\omega_{3} t}+2 A_{23}\left(k_{2}+k_{3}\right)
\end{aligned}
$$

$$
\begin{aligned}
& \cdot \mathrm{e}^{k_{2} x-\omega_{2} t} \mathrm{e}^{k_{3} x-\omega_{3} t}+2 A_{12} A_{13} A_{23}\left(k_{1}+k_{2}+k_{3}\right) \\
\cdot & \mathrm{e}^{k_{1} x-\omega_{1} t} \mathrm{e}^{k_{2} x-\omega_{2} t} \mathrm{e}^{k_{3} x-\omega_{3} t} \\
q & =1+\mathrm{e}^{k_{1} x-\omega_{1} t}+\mathrm{e}^{k_{2} x-\omega_{2} t}+\mathrm{e}^{k_{3} x-\omega_{3} t} \\
& +A_{12} \mathrm{e}^{k_{1} x-\omega_{1} t} \mathrm{e}^{-\omega_{2} t+k_{2} x}+A_{13} \mathrm{e}^{k_{1} x-\omega_{1} t} \mathrm{e}^{k_{3} x-\omega_{3} t} \\
& +A_{23} \mathrm{e}^{k_{2} x-\omega_{2} t} \mathrm{e}^{k_{3} x-\omega_{3} t} \\
& +A_{12} A_{13} \mathrm{~A}_{23} \mathrm{e}^{k_{1} x-\omega_{1} t} \mathrm{e}^{k_{2} x-\omega_{2} t} \mathrm{e}^{k_{3} x-\omega_{3} t} .
\end{aligned}
$$

Now, applying the MEFS yields

$$
\begin{aligned}
& A_{12}=\frac{5 b k_{1}{ }^{4}-15 b k_{1}^{3} k_{2}+20 b k_{1}{ }^{2} k_{2}{ }^{2}-15 b k_{1} k_{2}{ }^{3}+5 b k_{2}{ }^{4}+3 a k_{1}{ }^{2}-6 a k_{1} k_{2}+3 a k_{2}{ }^{2}}{5 b k_{1}{ }^{4}+15 b k_{1}{ }^{3} k_{2}+20 b k_{1}{ }^{2} k_{2}{ }^{2}+15 b k_{1} k_{2}^{3}+5 b k_{2}^{4}+3 a k_{1}{ }^{2}+6 a k_{1} k_{2}+3 a k_{2}^{2}}, \\
& A_{13}=\frac{5 b k_{1}{ }^{4}-15 b k_{1}{ }^{3} k_{3}+20 b k_{1}{ }^{2} k_{3}{ }^{2}-15 b k_{1} k_{3}{ }^{3}+5 b k_{3}{ }^{4}+3 a k_{1}{ }^{2}-6 a k_{1} k_{3}+3 a k_{3}{ }^{2}}{5 b k_{1}{ }^{4}+15 b k_{1}{ }^{3} k_{3}+20 b k_{1}{ }^{2} k_{3}{ }^{2}+15 b k_{1} k_{3}^{3}+5 b k_{3}{ }^{4}+3 a k_{1}{ }^{2}+6 a k_{1} k_{3}+3 a k_{3}^{2}} \text {, } \\
& A_{23}=\frac{5 b k_{2}{ }^{4}-15 b k_{2}{ }^{3} k_{3}+20 b k_{2}{ }^{2} k_{3}{ }^{2}-15 b k_{2} k_{3}{ }^{3}+5 b k_{3}{ }^{4}+3 a k_{2}{ }^{2}-6 a k_{2} k_{3}+3 a k_{3}{ }^{2}}{5 b k_{2}{ }^{4}+15 b k_{2}{ }^{3} k_{3}+20 b k_{2}{ }^{2} k_{3}{ }^{2}+15 b k_{2} k_{3}{ }^{3}+5 b k_{3}{ }^{4}+3 a k_{2}{ }^{2}+6 a k_{2} k_{3}+3 a k_{3}{ }^{2}} \\
& \omega_{1}=\frac{k_{1}\left(5 b^{2} k_{1}^{4}+5 a b k_{1}^{2}+a^{2}\right)}{5 b} \text {, } \\
& \omega_{2}=\frac{k_{2}\left(5 b^{2} k_{2}{ }^{4}+5 a b k_{2}{ }^{2}+a^{2}\right)}{5 b}, \\
& \omega_{3}=\frac{k_{3}\left(5 b^{2} k_{3}{ }^{4}+5 a b k_{3}{ }^{2}+a^{2}\right)}{5 b} .
\end{aligned}
$$

2.2. Multiple Soliton Solutions of $p K d V$ Equation (2). In the present subsection, the multiple soliton solutions of $\mathrm{pKdV}$ equation are obtained through the MEFS.

2.2.1. One-Soliton Solution of (2). To obtain one-soliton solution, it is assumed

$$
\begin{aligned}
v(x, t) & =\frac{p}{q}, \\
p & =A_{1} \mathrm{e}^{\theta_{1}},
\end{aligned}
$$

$$
q=1+\mathrm{e}^{\theta_{1}}
$$

where $A_{1}$ is a constant and

$$
\theta_{1}=k_{1} x-\omega_{1} t
$$

and the dispersion relation is

$$
\omega_{1}=\epsilon k_{1}^{5}+k_{1}^{3} .
$$




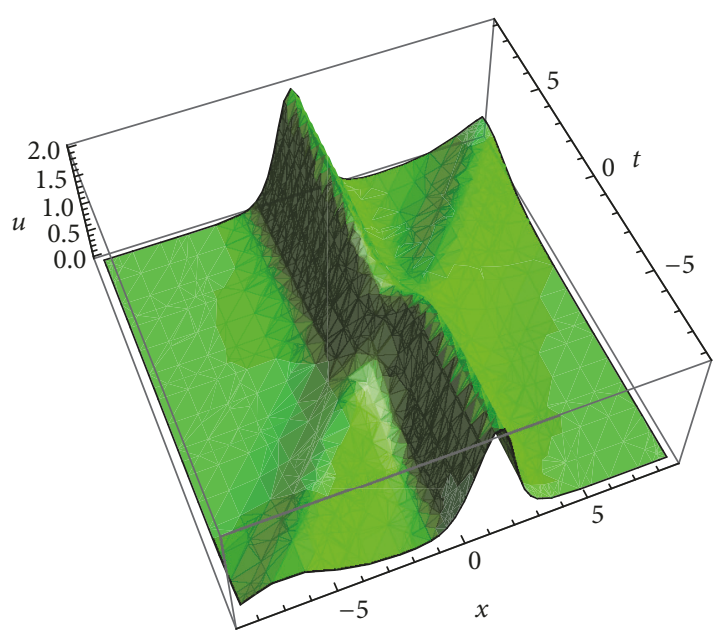

FIGURE 1: Evolution of the two-soliton solution (29).

Now, applying the MEFS results in

$$
A_{1}=2 k_{1}
$$

and, so, the resulting one-soliton solution can be presented as

$$
v(x, t)=\frac{2 k_{1} \mathrm{e}^{k_{1} x-\omega_{1} t}}{1+\mathrm{e}^{k_{1} x-\omega_{1} t}},
$$

where $k_{1}$ is arbitrary but $\omega_{1}$ is defined by (26).

2.2.2. Two-Soliton Solution of (2). To seek two-soliton solution, the following ansatz is considered

$$
v(x, t)=\frac{p}{q}
$$

in which $p$ and $q$ are defined as

$$
\begin{aligned}
p= & 2 k_{1} \mathrm{e}^{k_{1} x-\omega_{1} t}+2 k_{2} \mathrm{e}^{k_{2} x-\omega_{2} t} \\
& +2 A_{12}\left(k_{1}+k_{2}\right) \mathrm{e}^{k_{1} x-\omega_{1} t} \mathrm{e}^{k_{2} x-\omega_{2} t}, \\
q= & 1+\mathrm{e}^{k_{1} x-\omega_{1} t}+\mathrm{e}^{k_{2} x-\omega_{2} t}+A_{12} \mathrm{e}^{k_{1} x-\omega_{1} t} \mathrm{e}^{k_{2} x-\omega_{2} t} .
\end{aligned}
$$

Now, by applying the MEFS, we acquire

$$
\begin{aligned}
A_{12} & =\frac{k_{1}^{2}-2 k_{1} k_{2}+k_{2}^{2}}{k_{1}^{2}+2 k_{1} k_{2}+k_{2}^{2}}, \\
\omega_{1} & =\epsilon k_{1}^{5}+k_{1}^{3}, \\
\omega_{2} & =\epsilon k_{2}^{5}+k_{2}{ }^{3} .
\end{aligned}
$$

A profile of the evolution of the two-soliton solution (29) is given in Figure 1.

2.2.3. Three-Soliton Solution of (2). To derive three-soliton solution, it is assumed

$$
v(x, t)=\frac{p}{q}
$$

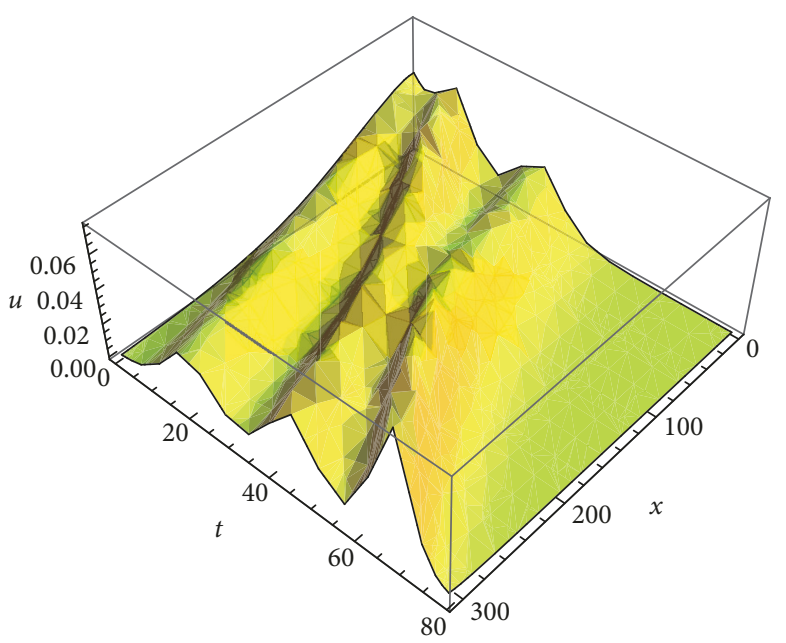

Figure 2: Evolution of the three-soliton solution (33).

in which $p$ and $q$ are defined as

$$
\begin{aligned}
p= & 2 k_{1} \mathrm{e}^{k_{1} x-\omega_{1} t}+2 k_{2} \mathrm{e}^{k_{2} x-\omega_{2} t}+2 k_{3} \mathrm{e}^{k_{3} x-\omega_{3} t} \\
& +2 A_{12}\left(k_{1}+k_{2}\right) \mathrm{e}^{-\omega_{1} t+k_{1} x} \mathrm{e}^{-\omega_{2} t+k_{2} x} \\
& +2 A_{13}\left(k_{1}+k_{3}\right) \mathrm{e}^{k_{1} x-\omega_{1} t} \mathrm{e}^{k_{3} x-\omega_{3} t}+2 A_{23}\left(k_{2}+k_{3}\right) \\
& \cdot \mathrm{e}^{k_{2} x-\omega_{2} t} \mathrm{e}^{k_{3} x-\omega_{3} t}+2 A_{12} A_{13} A_{23}\left(k_{1}+k_{2}+k_{3}\right) \\
& \cdot \mathrm{e}^{k_{1} x-\omega_{1} t} \mathrm{e}^{k_{2} x-\omega_{2} t} \mathrm{e}^{k_{3} x-\omega_{3} t}, \\
q & =1+\mathrm{e}^{k_{1} x-\omega_{1} t}+\mathrm{e}^{k_{2} x-\omega_{2} t}+\mathrm{e}^{k_{3} x-\omega_{3} t} \\
& +A_{12} \mathrm{e}^{k_{1} x-\omega_{1} t} \mathrm{e}^{-\omega_{2} t+k_{2} x}+A_{13} \mathrm{e}^{k_{1} x-\omega_{1} t} \mathrm{e}^{k_{3} x-\omega_{3} t} \\
& +A_{23} \mathrm{e}^{k_{2} x-\omega_{2} t} \mathrm{e}^{k_{3} x-\omega_{3} t} \\
& +A_{12} A_{13} A_{23} \mathrm{e}^{k_{1} x-\omega_{1} t} \mathrm{e}^{k_{2} x-\omega_{2} t} \mathrm{e}^{k_{3} x-\omega_{3} t}
\end{aligned}
$$

Now, applying the MEFS yields

$$
\begin{aligned}
A_{12} & =\frac{k_{1}^{2}-2 k_{1} k_{2}+k_{2}^{2}}{k_{1}^{2}+2 k_{1} k_{2}+k_{2}^{2}}, \\
A_{13} & =\frac{k_{1}^{2}-2 k_{1} k_{3}+k_{3}^{2}}{k_{1}^{2}+2 k_{1} k_{3}+k_{3}^{2}}, \\
A_{23} & =\frac{k_{2}^{2}-2 k_{2} k_{3}+k_{3}^{2}}{k_{2}{ }^{2}+2 k_{2} k_{3}+k_{3}^{2}}, \\
\omega_{1} & =\epsilon k_{1}^{5}+k_{1}{ }^{3}, \\
\omega_{2} & =\epsilon k_{2}{ }^{5}+k_{2}{ }^{3}, \\
\omega_{3} & =\epsilon k_{3}{ }^{5}+k_{3}{ }^{3} .
\end{aligned}
$$

A profile of the evolution of the three-soliton solution (33) is given in Figure 2. 


\section{Concluding Remarks}

We note that multiple soliton solutions of (1) and (2) are in agreement with [19, 21, 22]. Moreover, we should emphasize the approach employed here was independent of the bilinear forms, simplified Hirota method, Darboux transformation method, or Bell polynomial technique. Finally we can say the multiple exp-function algorithm is an elegant and versatile method that can be adopted to other NLEEs of mathematical physics.

\section{Data Availability}

The data used to support the findings of this study are included within the article.

\section{Conflicts of Interest}

The authors declare that they have no conflicts of interest.

\section{Acknowledgments}

This work of Qin Zhou was supported by the National Natural Science Foundation of China (Grant Nos. 11705130 and 1157149), this author was also sponsored by the Chutian Scholar Program of Hubei Government in China.

\section{References}

[1] A. W. Wazwaz, "Kinks and travelling wave solutions for Burgers-like equations," Applied Mathematics Letters, vol. 38, pp. 174-179, 2014.

[2] A.-M. Wazwaz, "A study on the $(2+1)$-dimensional and the (2+1)-dimensional higher-order Burgers equations," Applied Mathematics Letters, vol. 25, no. 10, pp. 1495-1499, 2012.

[3] X. B. Hu and Y. Li, "Backlund transformation and nonlinear superposition formula of DJKM equation," Acta Mathematica Scientia, vol. 11, no. 2, pp. 164-172, 1991.

[4] Y. Wang, H. Wang, and C. Temuer, "Lax pair, conservation laws, and multi-shock wave solutions of the DJKM equation with Bell polynomials and symbolic computation," Nonlinear Dynamics, vol. 78, no. 2, pp. 1101-1107, 2014.

[5] X. Lü and M. Peng, "Systematic construction of infinitely many conservation laws for certain nonlinear evolution equations in mathematical physics," Communications in Nonlinear Science and Numerical Simulation, vol. 18, no. 9, pp. 2304-2312, 2013.

[6] X. Lu, "Soliton behavior for a generalized mixed nonlinear Schrodinger model with N-fold Darboux transformation," Chaos, vol. 23, Article ID 033137, 2013.

[7] Y. Q. Yuan, B. Tian, L. Liu, X. Y. Wu, and Y. Sun, "Solitons for the $(2+1)$-dimensional Konopelchenko-Dubrovsky equations," Journal of Mathematical Analysis and Applications, vol. 460, pp. 476-486, 2018.

[8] X. Gao, "Mathematical view with observational/experimental consideration on certain $(2+1)$-dimensional waves in the cosmic/laboratory dusty plasmas," Applied Mathematics Letters, vol. 91, pp. 165-172, 2019.
[9] Z. Du, B. Tian, H. P. Chai, Y. Sun, and X. H. Zhao, "Rogue waves for the coupled variable-coefficient fourth-order nonlinear Schrdinger equations in an inhomogeneous optical fiber," Chaos, Solitons and Fractals, vol. 109, pp. 90-98, 2018.

[10] X. X. Du, B. Tian, X. Y. Wu, H. M. Yin, and C. R. Zhang, "Lie group analysis, analytic solutions and conservation laws of the $(3+1)$-dimensional Zakharov-Kuznetsov-Burgers equation in a collisionless magnetized electron-positron-ion plasma," The European Physical Journal Plus, vol. 133, p. 378, 2018.

[11] L. Liu, B. Tian, Y. Yuan, and Z. Du, "Dark-bright solitons and semirational rogue waves for the coupled Sasa-Satsuma equations," Physical Review E: Statistical, Nonlinear, and Soft Matter Physics, vol. 97, Article ID 052217, 2018.

[12] L. Liu, B. Tiana, Y. Q. Yuan, and Y. Sun, "Bright and dark Nsoliton solutions for the $(2+1)$-dimensional Maccari system," Eur. Phys. J. Plus, pp. 133-172, 2018.

[13] X. H. Zhao, B. Tian, X. Y. Xie, X. Y. Wu, Y. Sun, and Y. J. Guo, "Backlund transformation and Lax pair for a $(2+1)$-dimensional Davey-Stewartson system on surface waves of finite depth," Waves in Random and Complex Media, vol. 28, no. 2, pp. 356366, 2018.

[14] C. R. Zhang, B. Tian, X. Y. Wu, Y. Q. Yuan, and X. X. Du, "Rogue waves and solitons of the coherentlycoupled nonlinear Schrodinger equations with the positive coherent coupling," Physica Scripta, vol. 93, Article ID 095202, p. 11, 2018.

[15] X.-Y. Wu, B. Tian, L. Liu, and Y. Sun, "Rogue waves for a variable-coefficient Kadomtsev-Petviashvili equation in fluid mechanics," Computers \& Mathematics with Applications, vol. 76, no. 2, pp. 215-223, 2018.

[16] W. X. Ma, T. W. Huang, and Y. Zhang, "A multiple exp-function method for nonlinear differential equations and its application," Physica Scripta, vol. 82, Article ID 065003, 2010.

[17] W. X. Ma and Z. N. Zhu, "Solving the (3+1)-dimensional KP and BKP equations by the multiple exp-function algorithm," Applied Mathematics and Computation, vol. 218, pp. 11871-11879, 2012.

[18] R. Hirota and M. Ito, "Resonance of solitons in one dimension," Journal of the Physical Society of Japan, vol. 52, no. 3, pp. 744748,1983

[19] W. R. Shan, T. Z. Yan, X. Lu, M. Li, and B. Tian, "Analytic study on the Sawada-Kotera equation with a nonvanishing boundary condition in fluids," Communications in Nonlinear Science and Numerical Simulation, vol. 18, pp. 1568-1575, 2013.

[20] T. R. Marchant, "Numerical solitary wave interaction: the order of the inelastic effect," The ANZIAM Journal, vol. 44, no. 01, pp. 95-102, 2002.

[21] A. M. Wazwaz, "Multiple-soliton solutions of the perturbed $\mathrm{KdV}$ equation," Communications in Nonlinear Science and Numerical Simulation, vol. 15, no. 11, pp. 3270-3273, 2010.

[22] L. C. Liu, B. Tian, B. Qin, X. Lu, Z. Q. Lin, and W. J. Liu, "Backlund transformation, superposition formulae and Nsoliton solutions for the perturbed Korteweg-de Vries equation," Communications in Nonlinear Science and Numerical Simulation, vol. 17, pp. 2394-2402, 2012. 


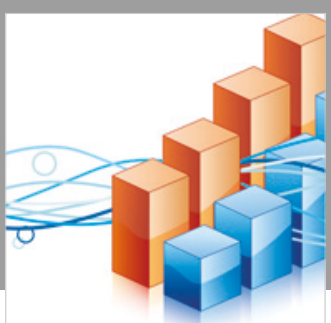

Advances in

Operations Research

\section{-n-m}
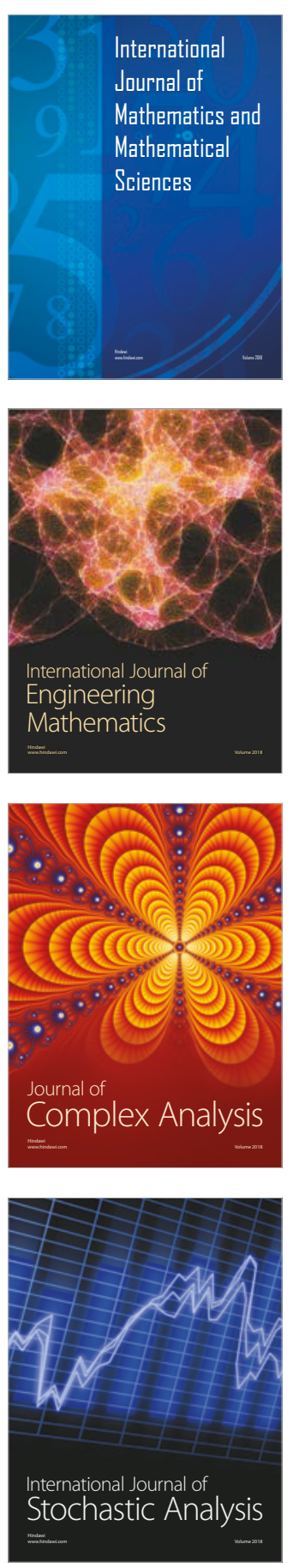
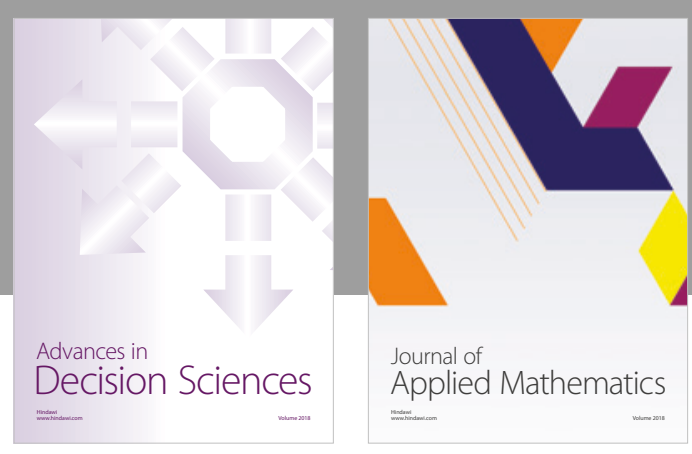

Journal of

Applied Mathematics
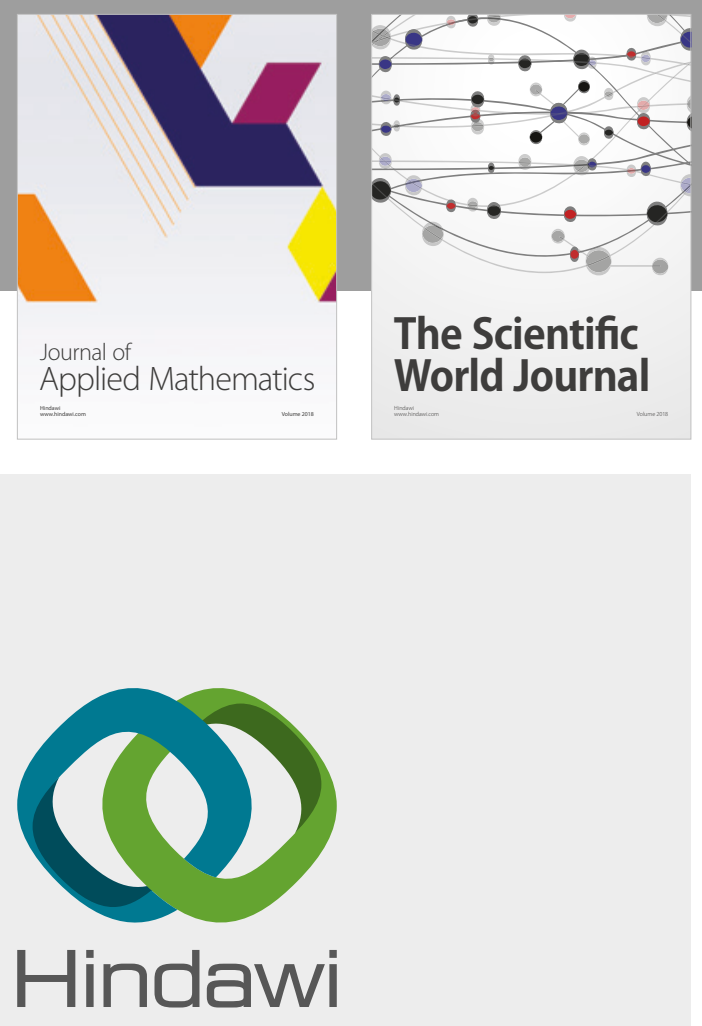

Submit your manuscripts at

www.hindawi.com

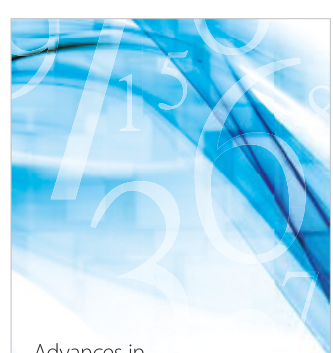

Advances in
Numerical Analysis
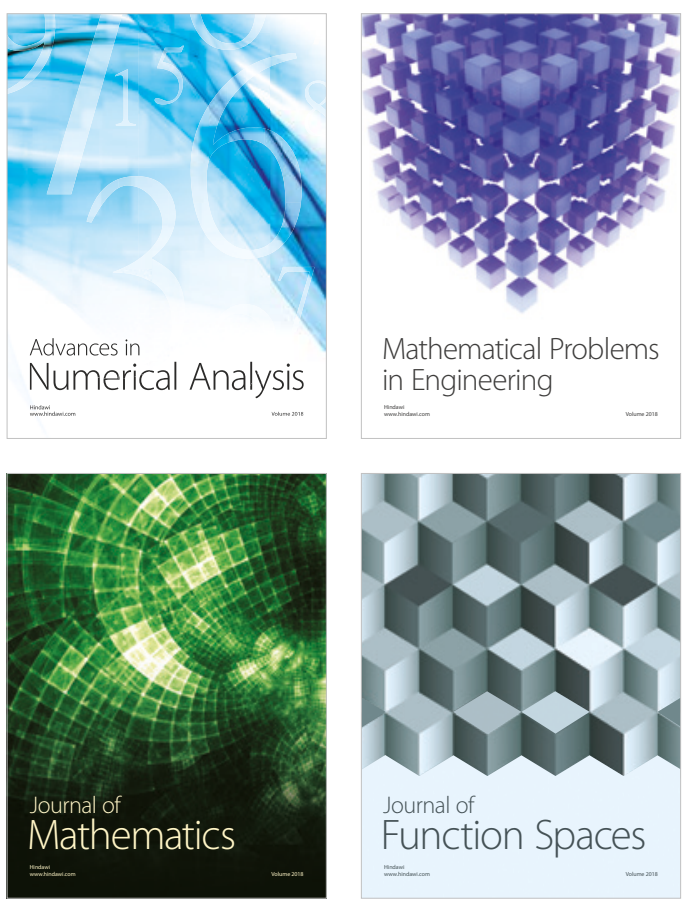

Mathematical Problems in Engineering

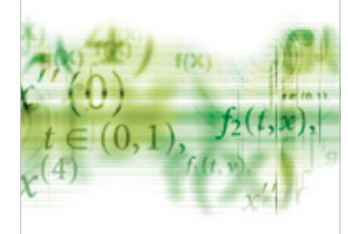

International Journal of

Differential Equations

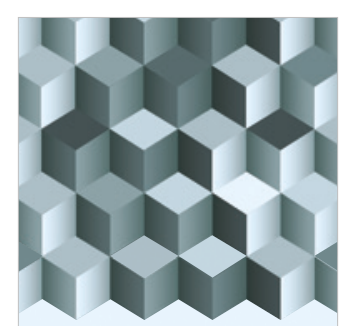

Journal of

Function Spaces

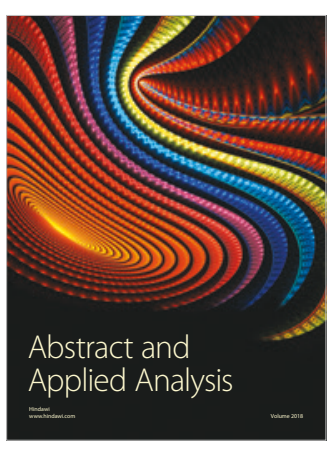

The Scientific

World Journal

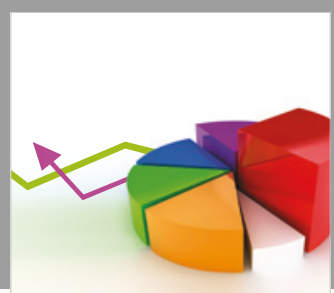

Journal of

Probability and Statistics
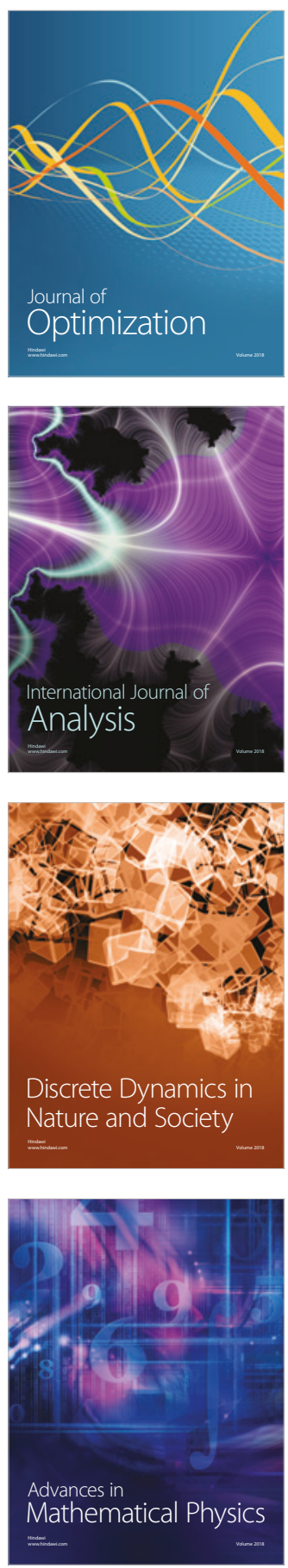